\title{
Timing in Orthodontics: A Simple Guide for the General Practitioner
}

\section{Elena Rodriguez}

Department of Orthodontics, University of Oviedo, Spain

\begin{abstract}
Thousands of children visit the dentist every year and even though general practitioners do their best to provide them with good oral health, there is always something that escapes our control: the way teeth and jaws are disposed of.

According to research carried out in the UK almost $55 \%$ of the children population has an orthodontic problem. So how should we general dentists know when it's the right timing to refer these patients for their first appointment with an orthodontist when even specialist's opinions on this matter differ?

These treatments, in general, are very time sensitive and if premature or delayed it leads to problems down the line which could have been prevented in the first place. The purpose of this study is to provide the general practitioner with the necessary tools and tricks to identify the most common skeletal malocclusions in the dental practice and to give them the ability to decide on the best moment to start with our orthodontic treatment based on age, development and the specific jaw problem.
\end{abstract}

Key Words: Oral health, Orthodontic treatment, Jaw problem, Children

\section{General Considerations}

A thorough background in craniofacial growth and development is necessary for every dentist. Even for those who end up never working with children, it can come in handy in order to comprehend conditions observed in adults and understanding the developmental processes that lead to these problems.

For those who do interact professionally with children and almost every dentist does so at least occasionally it is important to distinguish normal variation from the effects of abnormal or pathological processes. The timing of maturation and the potential to affect changes in the different facial planes of space is not uniform. Maxillary growth in the transverse plane of space, the first to cease growing, stops when the first bridging of the midpalatal suture begins, and not at final complete fusion [1-3].

Anteroposterior facial growth is most obvious in Class II and III skeletal malocclusions as both the maxilla and mandible move forward. Most accounts show these changes continuing until late adolescence, usually the mid-teen years and in some males until the late teens. This means that both treatment changes and failures to control growth can extend into the mid- to late-teen years and beyond. Vertical facial growth is the last to stop. Interestingly, this growth has been detected in both males and females into the third decade. This emphasizes why applying the correct timing for the respective problem is important. Palatal expansion is seemingly more urgent in earlier years, anteroposterior growth modification is more a mid growth activity, and vertical control requires a later approach if it can be accomplished.

\section{Oral Hygiene Control}

Orthodontic treatment with fixed appliances alters the oral environment, increases plaque accumulation, changes the composition of the flora, and complicates cleaning for the patient. Gingivitis and white spot lesions around fixed appliances are frequent side effects when preventive programs have not been implemented. The patients need more professional guidance in selecting the most appropriate oral health products and procedures for their individual needs and this is the dental professional's responsibility

\section{Back to Basics}

How does a child without any orthodontic problems look like? In early mixed dentition (7-8 years old) we can expect them to have minor indications of dental Class II malocclusions. This means that the molars are in a Class II relation and we have a bit of an overjet. This occurs due to the remanence of the leeway space resulting in wide jaws with a bit of crowding.

The next couple of years both jaws of the children begin to develop more in order to provide the necessary space to accommodate future teeth resulting in both maxillary and mandibular growth in proportion with the rest of the head.

At the end of the mixed dentition, the patient has to have wide arches with a proper form to provide enough room for the teeth while both upper and lower jaw should be in a skeletal class I relation. To ensure both forces are in balance the tongue has to have a high position by the palate working against the forces that are exerted by the perioral muscles. When we find ourselves diagnosing a 6-year-old child with an edge to edge bite, we should suspect a case of hidden skeletal Class III [4-6].

\section{Discovering the Imbalance}

One common reason for malocclusions stems from one or both jaws growing comparably more or less due to genetic factors. But also learned behaviors, that we might not be aware of, can negatively affect the growth of the jaws. If we add bad habits to an already present genetic condition the potential skeletal malocclusion only gets worse (Figure 1). 

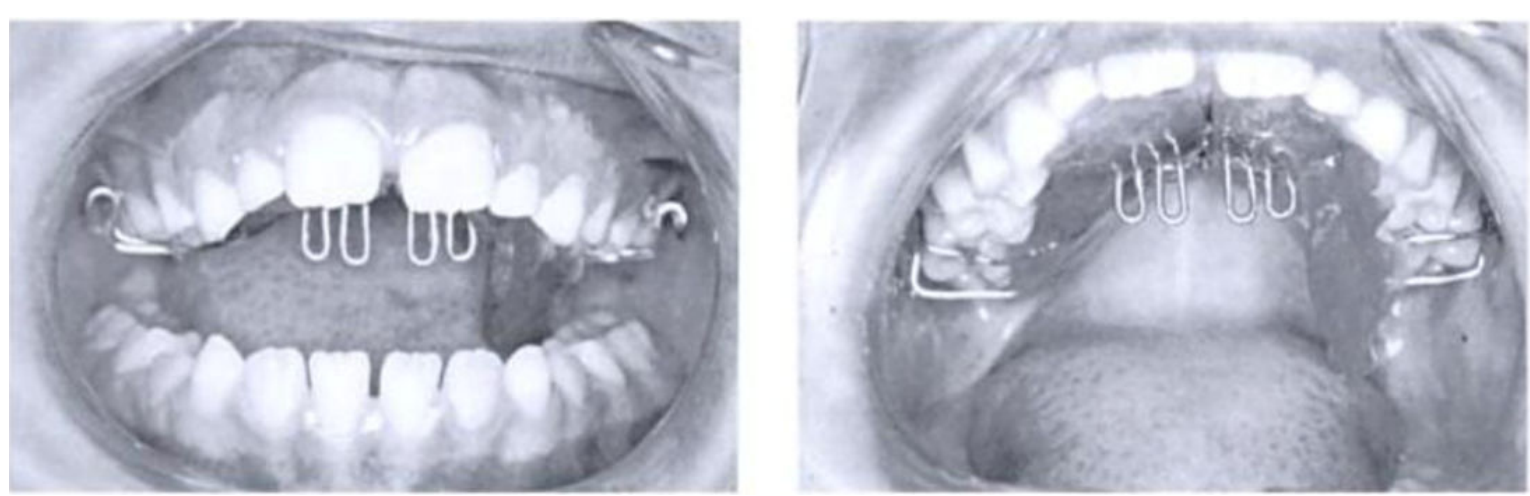

Figure 1. Tongue crib used for stopping with thumb sucking and tongue inter-position [7].

\section{Proceeding with the Orthodontic Treatment}

Here we first have to differentiate between the 3 different kinds of approaches (Figure 2).

Preventive approach: Monitoring the learned behaviors
Interceptive approach: Anterior or posterior crossbite, open bite

Orthopedic approach: These take place in childhood, puberty or youth

\begin{tabular}{|c|c|c|c|}
\hline Approaches & Childhood & Puberty & Youth \\
\hline Preventive approach & Monitoring habits & & \\
\hline Interceptive approach & Transversal problems & Sagital problems & Vertical problems \\
\hline Orthopedic approach & When needed & When needed & \\
\hline
\end{tabular}

Figure 2. Different approaches in the orthodontic treatment.

If there are grounds to believe that there's a behavior or a habit going on, we have to raise this issue with the patient/ parent.

In these cases, it's important that we refer our patients to the proper specialist before we begin with our treatment because its final result will be affected. But what are the most influential learned behaviors? Thumb-sucking in childhood, the interposition of the lip or tongue when swallowing or oral respiration. The latter can't really be considered a habit itself because sometimes children can't breathe through their noses because of an obstacle that closes the upper airway, such as septal deviation, turbinate and/or adenoidal hypertrophy.

In these cases, we will have to refer our patient to the ENT specialist and in some cases, the obstacle has to be surgically removed. If the oral breathing continues, the palate will not get the chance to develop. (Let's remember that the palate is the lower part of the nostrils) This will lead to the perioral muscles exerting forces on the maxillar which won't be compensated by the tongue that has now a lower positioneventually this will result in a maxillar compression. These oral breathers will have a typical long face because of the tongue position, open bite, posterior crossbite (with maxillar compression) and protrusion of incisors. If the patient underwent surgery, he will also be instructed to breathe normally through the nose. For the other behaviors such as interposition of the tongue, lip or thumb, the best thing is to convince the patient to stop. At the end of the treatment, if the habit is still going on, all of our efforts would have been for nothing. We can provide the patient with the correct tools such a visit to the speech therapist or we can also use different dental appliances like the tongue crib.

\section{Posterior Crossbite and the Urge of an Early Start}

The timing of maturation and the potential to effect a change in the different facial planes of space is not uniform. This usually means that by early adolescence palatal width increases would normally end and to mechanically alter this later with appliance therapy would require heavier forces. To minimize the risk of using heavy forces, the best way is to start a palatal expansion when 1.6 and the 2.6 erupt. This way the bones are finally relocated in the best place for them (Figure 3).

- We will avoid functional mandibulary deviations (that would lead to skeletal problems)

- We provide the bone with enough space for the future teeth 
- Easy treatment

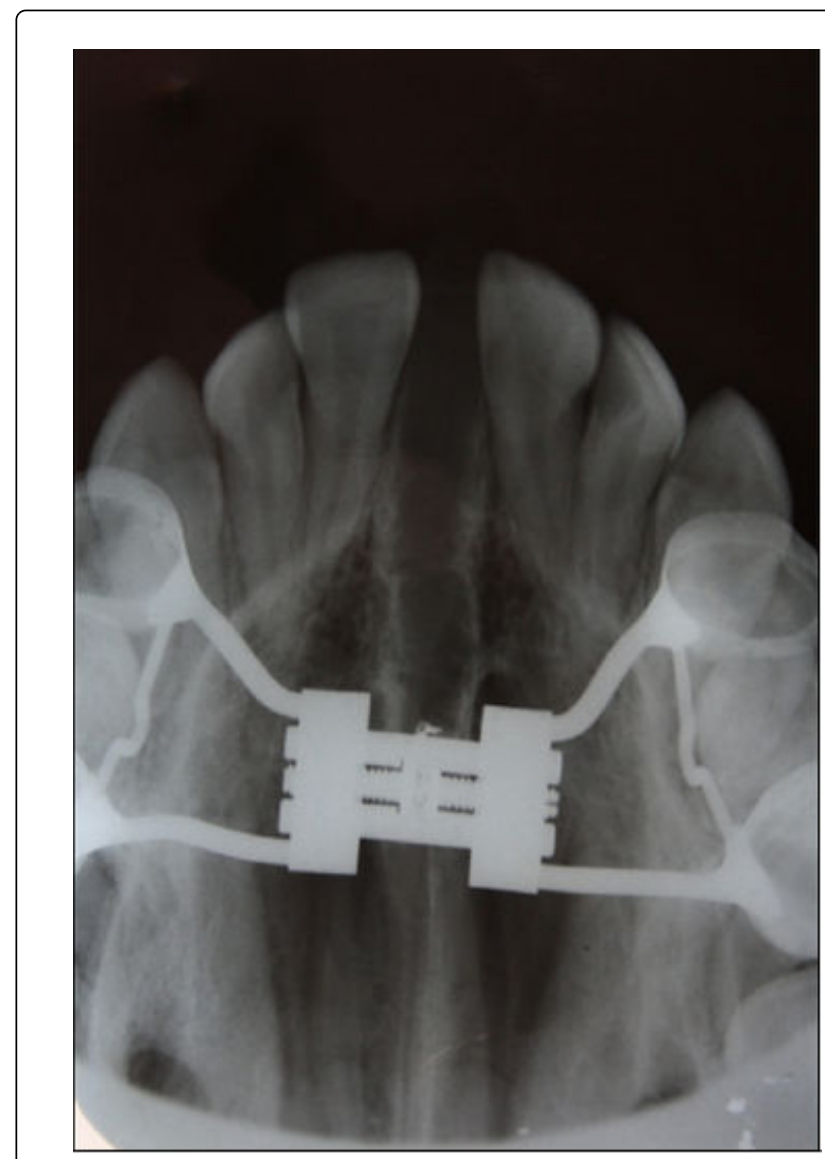

Figure 3. Radiographic examination of a palate expansion.

\section{Anterior Crossbite and Skeletal Class III}

What can we do in these cases? when we face a dental class III profile the best thing is to interfere as soon as possible because with time this will develop into a skeletal class III that can lead to the necessity a surgical procedure. In around $70 \%$ of the skeletal class III cases we see in our daily practice, the problem is the upper jaw. That means that we have a normal lower jaw but it doesn't have a natural obstacle that prevents it from growing further. If left untreated, we will end up a concave profile over the years that are very difficult to improve without surgery. So if we encounter a 6-year-old child with an edge to edge bite, the best is to refer this patient to the orthodontist.

What do we do as orthodontists? Depending on the jaw affected we will have to think about a statement treatment working by goals. There is no doubt that maxillary protraction at an early age usually produces clinical improvement in a skeletal Class III patient. Important concerns are the extent to which this will be maintained long-term and the chance that orthognathic surgery eventually will be necessary despite the early treatment. The treatment of choice is the rapid expansion (bonded or banded) associated with a facemask to achieve maxillary protraction. Children who have skeletal Class III malocclusion because of excessive growth of the mandible are extremely difficult to treat. Modifying true mandibular prognathism is a difficult task, regardless of the chosen method. This often leads to irrational choices by practitioners and parents in attempts to control crossbite and chin prominence as the child grows and to avoid surgical treatment when the child has matured. When our patient has an anterior crossbite, we will have to handle the lower jaw to try to retract it to centric relation. If the $\mathrm{CR}$ is the same as our normal intercuspidation, we will have to suspect of a skeletal problem, if it's not the same, then we are facing a dental class III in which both jaws have a normal dimension but due to a disturbing tooth or the way the incisors grew, the patient can't bite normally. At any case, we have to act fast. The bones tend to adapt to the environmental situation and what in begin looked like a simple treatment, can lead to a surgical case.

\section{Growth Modification Regarding Skeletal Class II Treatments}

When we face a skeletal class II malocclusion, there is a tendency to believe that the sooner that the treatment gets started the better.

This popular belief led to cases in which the patients, who had achieved a stable occlusion before the adolescent growth spurt, suffered a skeletal discrepancy by the time they were fully developing.

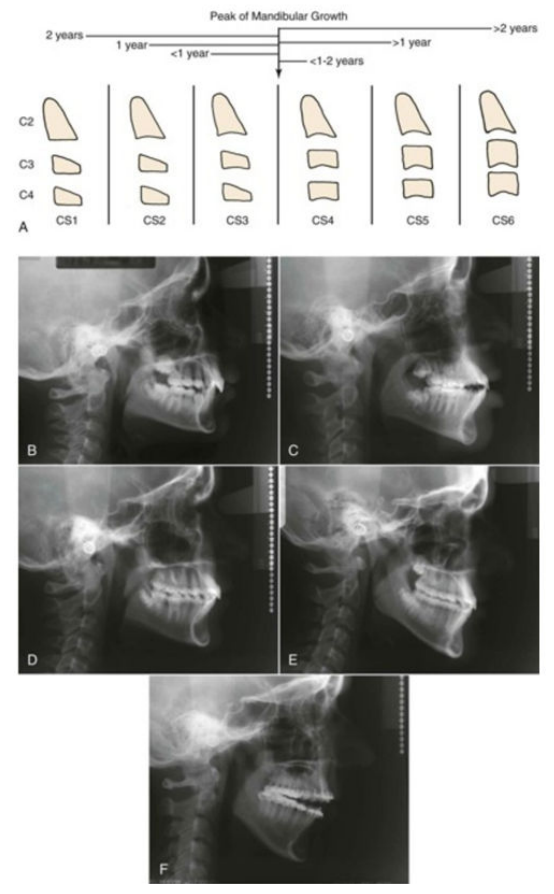

Figure 4. Vertebral ages calculated from the image of the cervical vertebrae seen in a lateral cephalometric radiograph [8].

Here was not taken into account that the growth occurs on different moments for the three planes of space.

During the primary dentition, the only plane of space in which a growth modification can be truly made is the transversal one. A bit of relapse is to be expected in any case because of the genetical component that makes growth take place in the disproportionate pattern.

When the only problem being faced is the sagittal discrepancy, is better to wait until the adolescent growth spurt is getting closer. The final result will be the same as if the 
treatment had started before. This way we will avoid years of unnecessary treatments that would finally affect our patients' motivation and compliance with our orthodontic plan.

It might be logic to think that if the necessary treatment for sagittal discrepancy might have to take place near the growth peak, the possibility to wait for treating skeletal class III cases is also there. But let us not forget that in a huge percentage of the Class III cases the problem is the upper jaw and that in these cases there is also a transversal stimulation needed on this bone. An early approach would be key then.

However, a child with jaw discrepancy can benefit from treatment during the preadolescent years when esthetics and psychosocial problems are present.

Dental and skeletal profile cases that can lead to dental trauma due to increased overjet are also susceptible to early treatment.

Nonetheless, it is probably prudent to consider reducing overjet for the most accident-prone children. For each patient, the benefits of early treatment must be considered against the risk and cost of prolonging the total treatment period. How one evaluates the growth stages and timing appears to make a difference, and different methods have advocates and detractors, based on the assessment approach. The cervical vertebral maturation staging (CVMS) method related to mandibular growth changes may yield different results than a timing based on hand-wrist radiographic estimation of skeletal maturation. In fact, differences of opinion exist on the appropriateness of each technique and even on how to apply the CVMS method. It may be that the most reliable, valid, and critical use of the CVMS method is differentiating the pre mandibular from post mandibular growth peak phases. Given the reduced radiation (because the images are available as part of the cephalometric radiograph), simplicity in learning, and excellent accuracy of the CVMS method among nonradiologist growth assessors like dentists and orthodontists, this method has a strong appeal and is certain to evolve. Once the growth ceases, we have to focus on compensatory treatments or monitor the patients till it's a good time for the surgery. In the following figure the different growth stages can be seen (Figure 4).

- A. Diagrammatic drawings and descriptions of the stages

- B. Stage 2, indicating that peak growth at adolescence is still a year or so ahead

- C. Stage 3, which on average is less than 1 year prior to peak growth. D Stage 4, typically a year or so beyond peak growth

- E. Stage 5, more than 1 year beyond the peak of the growth spurt, probably with more vertical than anteroposterior growth remaining

- F. Stage 6, more than 2 years beyond peak growth (but in a patient with a severe skeletal problem, especially excessive mandibular growth, not necessarily ready for a surgery-the best way to determine the cessation of growth is serial cephalometric radiographs)

\section{Conclusion}

Every skeletal malocclusion needs a different timing based on the origin, location, and age. The timing of maturation and the potential to affect changes in the different facial planes of space is not uniform.

\begin{tabular}{|c|c|c|}
\hline Problem & Early mixed dentition (Age 6-7) & $\begin{array}{l}\text { Close to growth spurt (Age } \\
\text { 12-13) }\end{array}$ \\
\hline Posterior crossbite & Refer to orthodontist & $\begin{array}{l}\text { Might already be moderate } \\
\text { skeletal condition }\end{array}$ \\
\hline Anterior crossbite & Refer to orthodontist & $\begin{array}{l}\text { Might already be moderate } \\
\text { skeletal condition }\end{array}$ \\
\hline $\begin{array}{l}\text { Anterior crossbite with skeletal } \\
\text { class III }\end{array}$ & $\begin{array}{l}\text { Refer to orthodontist inmediately } \\
\text { (can lead to severe skeletal class } \\
\text { III) }\end{array}$ & $\begin{array}{l}\text { Might already be severe skeletal } \\
\text { condition }\end{array}$ \\
\hline Skeletal class II without habits & & Re-evaluate \\
\hline Skeletal class II with habits & Monitor behavior & Might need orhodontic treatment \\
\hline
\end{tabular}

Figure 5. Overview of the malocclusion based on age and starting moment.

Maxillary growth in the transverse plane of space stops when the first bridging of the midpalatal suture begins, and not at final complete fusion. Anteroposterior facial growth changes until late adolescence, usually the mid-teen years and in some males until the late teens. This means that both treatment changes and failures to control growth can extend into the mid- to late-teen years and beyond.

Vertical facial growth is the last to stop. This growth has been detected in both males and females into the third decade.
When our malocclusion includes a variation in the three planes of space we have to intercede as soon as possible. Pathologic changes in the transverse plane would be the first to treat by the moment we see the malocclusion.

Sagittal abnormalities would be the next to treat. Depending on the jaw affected and the Angle class that we have we would proceed sooner or later. In the end, our treatments would be based on the prevention of an upcoming 
malocclusion and interception when the pathological variation is already taking place (Figure 5).

\section{References}

1. Bravo González LA. Manual de ortodoncia. 2003

2. Rodrigues de Almeida R. Early treatment protocol for skeletal class III maloclussion. Brazilian Dental Journal. 2013; 24: 2.

3. Musich D, Busch MJ. Early orthodontic treatment: Current clinical perspectives. Alpha Omegan. 2007; 100: 17-24.

4. Sim JM. The twelve rules of orthodontic treatment during mixed dentition. Journal of general orthodontics. 1998; 9: 22-27.
5. Harrison JE. Orthodontic treatment for prominent upper front teeth in children. The Cochrane database of systematic reviews. 2007; 18: CD003452.

6. Quintero AM, García C. Control de la hygiene oral en los pacientes con ortodoncia. Revista Nacional de Odontología. 2013; 9: $37-45$.

7. Canut Brusola JA. Ortodoncia clínica y terapéutica. 2000; 2: 289.

8. Proffit WR. Contemporary orthodontics. 2008; 5: 82. 